\title{
On the local geometry of definably stratified sets
}

\author{
David Trotman and Guillaume Valette
}

\begin{abstract}
We prove that a theorem of Pawłucki, showing that Whitney regularity for a subanalytic set with a smooth singular locus of codimension one implies the set is a finite union of $C^{1}$ manifolds with boundary, applies to definable sets in polynomially bounded o-minimal structures. We give a refined version of Pawłucki's theorem for arbitrary o-minimal structures, replacing Whitney (b)-regularity by a quantified version, and we prove related results concerning normal cones and continuity of the density. We analyse two counterexamples to the extension of Pawłucki's theorem to definable subsets in general o-minimal structures, and to several other statements valid for subanalytic sets. In particular we give the first example of a Whitney $(b)$-regular definably stratified set such that the density is not continuous along a stratum.
\end{abstract}

\section{Introduction}

We recall the statement of a striking and useful theorem of W. Pawłucki.

Theorem 1.1. (Pawtucki $[\mathbf{P a})$ A subanalytic stratified set $S$ with a smooth singular locus $L$ of codimension 1 is Whitney $(b)$-regular if and only if $S$ is a finite union of $C^{1}$ manifolds-with-boundary $S_{1}, \ldots, S_{k}$ each of which has boundary $L$.

Pawłucki used this theorem to prove a version of Stokes' theorem for subanalytic sets, later generalised by Łojasiewicz $\overline{\mathbf{L o j}}$ and by Valette $\mathbf{V a 4}$. It has also been used in various ways in papers of Bernig $[\mathbf{B e}$, Chossat-Koenig $[\mathbf{C K}]$, Comte $\mathbf{C}$, Jeddi $[\mathbf{J}$, Orro-Pelletier $[\mathbf{O P}$ and Valette $[\mathbf{V a 3}$, among others.

It is natural to consider possible generalisations of Pawłucki's theorem to definable sets in o-minimal structures. We prove in section 3 that Pawłucki's theorem applies to definable sets in polynomially bounded o-minimal structures (Corollary 3.11). Furthermore we give in Corollary 3.10 a refined version of Pawłucki's theorem

2010 Mathematics Subject Classification. Primary 03C64, 32S15, 53B25, 58A35; Secondary 32S60 .

Visits to the Jagiellonian University in Krakow and the University of Provence in Marseille, during which this work developed, were supported by a PHC Polonium project. The research began while both authors were resident at the Fields Institute in Toronto during the thematic program, "O-minimal Structures and Real Analytic Geometry". We gratefully acknowledge the support of the French Ministry of Foreign and European Affairs (MAEE), the French Ministry of Higher Education and Research (MESR), the Fields Institute and the French Embassy in Ottawa.

(C) 0000 (copyright holder) 
which applies to arbitrary o-minimal structures, replacing Whitney (b)-regularity by a quantified version first described by the second author in his thesis Va1 (directed by the first author). We also generalise a theorem of Hironaka $[\mathbf{H}]$ stating that Whitney regularity of an analytic variety controls the normal cone structure, and a theorem of Comte $[\mathbf{C}$ stating that Kuo's ratio test ensures continuity of the density for subanalytic sets.

In section 4 we analyse two counterexamples to the natural generalisation of Pawłucki's theorem for definable subsets in o-minimal structures which are not polynomially bounded. These also provide counterexamples to several classical results describing relations between well-known equisingularity conditions for subanalytic strata, including the above theorem of Hironaka and a result of Navarro Aznar and the first author [NT]. We also obtain the first example of a Whitney $(b)$-regular definable stratified set for which the density is not continuous along each stratum; note that the density is continuous along strata of a $(b)$-regular definable stratified set when the o-minimal structure is polynomially bounded [Va2], $\mathbf{N V}$.

\section{Definitions.}

Let $k \geq 2$ be an integer. Let $S$ be a closed stratified subset of $\mathbf{R}^{n}$, such that the strata are differentiable submanifolds of class $C^{k}$. For each stratum $X$ of $S$ denote by $C_{X} S$ the normal cone of $S$ along $X$, i.e. the restriction to $X$ of the closure of the set $\{(x, \mu(x \pi(x))): x \in S \backslash X\} \subset \mathbf{R}^{n} \times S^{n-1}$, where $\pi$ is the local canonical retraction onto $X$ (recall that this is defined on a neighbourhood of a differentiable manifold of class $C^{2}$ by taking the unique nearest point on $\left.X\right), \mu(x)$ is the unit vector $\frac{x}{\|x\|}$, and $u v$ denotes the vector $v-u$, where $u$ and $v$ are points of $\mathbf{R}^{n}$. In fact $C_{X} S$ is a union of normal cones $C_{X} Y_{i}$, where the $\left\{Y_{i}\right\}$ are the strata of $S$ whose closures contain $X$. The following two properties of the normal cone express that $S$ behaves well along the stratum $X$ (at the point $x$ ).

Definition 2.1. Condition (n): The fibre $\left(C_{X} S\right)_{x}$ of $C_{X} S$ at a point $x$ of $X$ equals the tangent cone $C_{x}\left(S_{x}\right)$ to the fibre $S_{x}=S \cap \pi^{-1}(x)$ of $S$ at $x$.

Definition 2.2. Normal pseudo-flatness $(n p f)[\mathbf{H}]$ : The stratified set $S$ is said to be normally pseudo-flat along $X$ when the projection $p: C_{X} S \rightarrow X$ is open.

When a stratification satisfies simultaneously two regularity conditions, say Whitney $(a)$-regularity and $(n)$, we write that it is $(a+n)$-regular. Subanalytic stratifications satisfying $(a+n)$ or $(n p f)$ have normal cones with good behaviour from the point of view of the dimension of the fibres. In fact they satisfy the condition

$$
\operatorname{dim}\left(C_{X} S\right)_{x} \leq \operatorname{dim} S-\operatorname{dim} X-1 . \quad(*)
$$

This is obvious for $(a+n)$, while for $(n p f)$ it follows from [T2]. For differentiable stratifications one may not always be able to define the dimension. Despite the bound (*), the tangent cone $C_{x}\left(S_{x}\right)$ to the fibre $S_{x}=S \cap \pi^{-1}(x)$ (hence the fibre $\left(C_{X} S\right)_{x}$ of the normal cone, assuming $\left.(n)\right)$ can be quite arbitrary : work of Ferrarotti, Fortuna and Wilson [FFW] shows that each closed semi-algebraic cone of codimension $\geq 1$ is realised as the tangent cone at a point of a certain real algebraic variety, while Kwieciński and the first author showed that every closed 
cone is realised as the tangent cone at an isolated singularity of a certain $C^{\infty}$ (b)-regular stratified set $\mathbf{K T}$.

Hironaka proved in $[\mathbf{H}$ that Whitney $(b)$-regular stratifications of any analytic set (real or complex) satisfy condition $(n)$ and are normally pseudo-flat along each stratum. Twenty years later Henry et Merle HM2 obtained $(n)$ with $S$ replaced by $X \cup Y$ when $X$ and $Y$ are two adjacent strata of a sub-analytic Whitney stratification of $X \cup Y$. In OT3 Orro and the first author introduced a metric invariant of Kuo's ratio test $(r)$ (introduced by Kuo $[\mathbf{K}]$ in 1971), denoted by $\left(r^{e}\right)$, for $e \in[0,1]$. Every $C^{2}(w)$-regular stratification satisfies automatically $(a)$ and $\left(r^{e}\right)$, i.e. $\left(a+r^{e}\right)$. Here as usual $(w)$ refers to the Kuo-Verdier condition Ve which implies trivially Kuo's ratio test $(r)$. Recall that $(r)$ implies Whitney $(b)$-regularity for subanalytic strata $\mathbf{K}$. In Proposition 3.2 below we show that $(r)$ implies $(b)$ for definable sets in any o-minimal structure, showing that $(w)$ also implies $(b)$. This is not the case for general stratified sets. For subanalytic strata it was observed in OT3 that the combination $\left(a+r^{e}\right)$ is equivalent to Kuo's ratio test $(r)$, and the proof goes through without difficulty for definable stratifications in polynomially bounded ominimal structures; by [T1 we know that $(r)$ is strictly weaker than $(w)$ in the

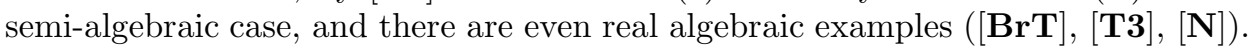

The equivalence of $(b),(r)$ and $(w)$ for complex analytic stratifications is a consequence of Teissier's programme of characterising $(b)$-regularity by equimultiplicity of polar varieties, completed in 1982 ([Te1], HM1]). One can consult [Te2] and Me for detailed surveys of the complex equisingularity theory.

In OT2 it was proved that every $\left(a+r^{e}\right)$-regular stratification is normally pseudo-flat and satisfies condition $(n)$, without subanalyticity. Hence for $(r)$-regular stratifications which are definable in a polynomially bounded o-minimal structure, $(n)$ and $(n p f)$ hold. We shall show in section 4 that this is not the case for o-minimal structures which are not polynomially bounded.

We recall below, for the convenience of the reader, the definitions of the conditions $(a)$ and $(b)$ of Whitney, $(r)$ of Kuo $[\mathbf{K}],\left(r^{e}\right)$ of Orro-Trotman and $(w)$ of Kuo-Verdier $\mathbf{V e}$. We also recall Teissier's notion of $\left(E^{\star}\right)$ where $(E)$ is any equisingularity criterion.

Let $X$ and $Y$ be two submanifolds of $R^{n}$ such that $X \subset \operatorname{cl}(Y)$, and let $\pi$ be a local retraction onto $X$. Following Hironaka $[\mathbf{H}]$, we denote by $\alpha_{Y, X}(y)$ the distance of $T_{y} Y$ to $T_{\pi(y)} X$, which is

$$
\alpha_{Y, X}(y)=\max \left\{<\mu(u), \mu(v)>: u \in N_{y} Y \backslash\{0\}, v \in T_{\pi(y)} X\right\},
$$

and we denote by $\beta_{Y, X}(y)$ the angle of $y \pi(y)$ to $T_{y} Y$ expressed as

$$
\left.\beta_{Y, X}(y)=\max \{<\mu(u), \mu(y \pi(y))\}>: u \in N_{y} Y \backslash\{0\}\right\},
$$

where $<,>$ is the scalar product on $R^{n}$. For $v \in R^{n}$, the distance of the vector $v$ to a plane $B$ is

$$
\eta(v, B)=\sup \left\{<v, n>: n \in B^{\perp},\|n\|=1\right\} .
$$

Set

$$
d(A, B)=\sup \{\eta(v, B): v \in A,\|v\|=1\},
$$

so that in particular $\alpha_{Y, X}(y)=d\left(T_{\pi(y)} X, T_{y} Y\right)$. Set also 


$$
R_{Y, X}(y)=\frac{\|y\| \alpha_{Y, X}(y)}{\|y \pi(y)\|} \quad \text { and } \quad W_{Y, X}(y, x)=\frac{d\left(T_{x} X, T_{y} Y\right)}{\|y x\|} .
$$

Definition 2.3. The pair of strata $(X, Y)$ satisfies, at $0 \in X$ :

condition (a) if, for $y$ in $Y$,

$$
\lim _{y \rightarrow 0} \alpha_{Y, X}(y)=0,
$$

condition $\left(b^{\pi}\right)$ if, for $y$ in $Y$,

$$
\lim _{y \rightarrow 0} \beta_{Y, X}(y)=0 .
$$

condition (b) if, for $y$ in $Y$,

$$
\lim _{y \rightarrow 0} \alpha_{Y, X}(y)=\lim _{y \rightarrow 0} \beta_{Y, X}(y)=0,
$$

condition $(r)$ if, for $y$ in $Y$,

$$
\lim _{y \rightarrow 0} R_{Y, X}(y)=0,
$$

condition $(w)$ if, for $y$ in $Y$ and $x$ in $X, W_{Y, X}(y, x)$ is bounded near 0 .

In OT2 P. Orro and the first author introduced the following condition of Kuo-Verdier type.

Definition 2.4. Let $e \in[0,1)$. One says that $(X, Y)$ satisfies condition $\left(r^{e}\right)$ at $0 \in X$ if, for $y \in Y$, the quantity

is bounded near 0 .

$$
R^{e}(y)=\frac{\|\pi(y)\|^{e} \alpha_{Y, X}(y)}{\|y \pi(y)\|} .
$$

This condition is a $C^{2}$ diffeomorphism invariant. It is Verdier's condition $(w)$ when $e=0$, hence $(w)$ implies $\left(r^{e}\right)$ for all $e \in[0,1)$. But, unlike $(w)$, condition $\left(r^{e}\right)$ when $e>0$ does not imply condition $(a)$ : a counter-example which is a semialgebraic surface can be obtained by pinching a half-plane $\{z \geq 0, y=0\}$ of $\mathbf{R}^{3}$, with boundary the $x$-axis $X$ in a cuspidal region $\Gamma=\left\{x^{2}+y^{2}<z^{p}\right\}$, where $p$ is an odd integer such that $p>\frac{2}{e}$, such that in $\Gamma$ there are sequences tending to 0 for which condition $(a)$ fails. Such an example will be $\left(r^{e}\right)$-regular.

TheOREM 2.5. OT3. Every $C^{2}\left(a+r^{e}\right)$-regular stratification is normally pseudo-flat and satisfies condition $(n)$.

Corollary 2.6. For $C^{2}(r)$-regular stratifications which are definable in a polynomially bounded o-minimal structure, $(n)$ and $(n p f)$ hold.

In section 4 we give a counterexample to the corollary for any non-polynomially bounded o-minimal structure.

Now we recall the definition of $E^{*}$-regularity for $E$ an equisingularity condition, as in OT1. This notion came from the discussion of B. Teissier in his 1974 Arcata lectures [Te. Teissier stated that one requirement for an equisingularity condition to be good is that it be preserved after intersection with generic linear spaces containing a given linear stratum. Various equisingularity conditions have been 
shown to have this property, notably Whitney $(b)$-regularity for complex analytic stratifications ([Te1], HM1]), and Kuo's ratio test $(r)$, the Kuo-Verdier condition $(w)$ [NT, and Mostowski's $(L)$-regularity for subanalytic stratifications [JTV] .

Definition 2.7. Let $M$ be a $C^{2}$-manifold. Let $X$ be a $C^{2}$-submanifold of $M$ and $x \in X$. Let $Y$ be a $C^{2}$-submanifold of $M$ such that $x \in Y$, and $X \cap Y=\emptyset$. Let $E$ denote an equisingularity condition (for example $(b),(r)$ or $(w)$ ). Then $(X, Y)$ is said to be $E_{\operatorname{cod} k}$-regular at $x(0<k<\operatorname{cod} X)$ if there is an open dense subset $U_{k}$ of the Grassmann manifold of codimension $k$ subspaces of $T_{x} M$ containing $T_{x} X$ such that if $W$ is a $C^{2}$ submanifold of $M$ with $X \subset W$ near $x$, and $T_{x} W \in U_{k}$, then $W$ is transverse to $Y$ near $x$, and $(X, Y \cap W)$ is E-regular at $x$.

Definition 2.8. $(X, Y)$ is said to be $E^{*}$-regular at $x$ if $(X, Y)$ is $E_{\operatorname{cod} k}$-regular for all $k, 0<k<\operatorname{cod} X$.

Theorem 2.9. [NT] For subanalytic stratifications, $(r)$ implies $\left(r^{*}\right)$ and $(w)$ implies $\left(w^{*}\right)$.

In this sub-analytic case, because $(b)$ implies $(r)$ over 1-dimensional strata, and $(r)$ always implies $(b)$, we deduce the following corollary.

Corollary 2.10. [NT] For subanalytic (b)-regular stratifications, $\left(b^{*}\right)$ holds over every 1-dimensional stratum.

In section 4 we give counterexamples to the above Theorem and Corollary in any non-polynomially bounded o-minimal structure.

\section{Normal pseudo-flatness in the non-polynomially bounded case}

In this section we give several theorems indicating for which o-minimal structures the definably stratified sets have similar properties to sub-analytic sets, thus avoiding the pathologies exhibited by the functions $f$ and $g$ described in section 4 . These theorems are based on earlier work of the second author in Va1.

In the final section of this paper we shall show that condition $(r)$ is too weak to ensure normal pseudo-flatness in non-polynomially bounded o-minimal structures. In this section we explain how to overcome this problem: we introduce a slightly stronger condition which is enough to entail normal pseudo-flatness. We will also show that the density is continuous along the strata of a stratification satisfying this condition.

We prove in the following proposition (3.1) that one can extend the result of Kuo [ $\mathbf{K}$ for semianalytic stratifications that $(b)$ implies $(r)$ along strata of dimension one (extended by Verdier [Ve to subanalytic stratifications), to all stratified sets definable in polynomially bounded o-minimal structures. The result of Proposition 3.1 is sharp: the example in section 4 below is the first case known of an o-minimal structure with a $(b)$-regular definable stratification which does not satisfy Kuo's ratio test $(r)$. Moreover by Miller's dichotomy $[\mathbf{M}]$ every non polynomially bounded o-minimal structure has such an example.

Recall that Kuo's ratio test $(r)$ holds for $(W, X)$ at $(0,0,0)$ if and only if

$$
R(x, y, z)=\frac{|(x, y, z)| . \delta\left(T_{(x, 0,0)} X, T_{(x, y, z)} W\right)}{|(y, z)|} \rightarrow 0
$$


as $(x, y, z)$ tends to $(0,0,0)$ on $W$. For $E$ and $F$ vector subspaces of $\mathbb{R}^{n}$, the function $\delta$ is defined by:

$$
\delta(E, F):=\sup _{|u|=1, u \in E} d(u, F) .
$$

Proposition 3.1. Let $X$ and $Y$ be $C^{2}$ disjoint submanifolds which are definable in a polynomially bounded o-minimal structure with $\operatorname{dim} X=1$, and $0 \in X \cap \operatorname{cl}(Y)$. If the pair $(Y, X)$ satisfies the Whitney (b) condition then it also fulfills Kuo's ratio test $(r)$.

Proof. Take such $(Y, X)$ satisfying Whitney's condition $(b)$ at $0 \in X$. After a local change of coordinates we may assume that $X$ is a line. We have to show that the Kuo ratio $R$ tends to zero along any definable arc $\gamma:(0, \varepsilon) \rightarrow Y$ with $\gamma(0)=0$. We may assume that $\gamma$ is parameterized by arc-length. We denote by $P_{t}$ the orthogonal projection onto $T_{\gamma(t)} Y^{\perp}$, and by $\pi$ the orthogonal projection onto $X$. As $(Y, X)$ is Whitney $(b)$ regular:

$$
\lim _{t \rightarrow 0} P_{t}\left(\frac{\gamma(t)-\pi((\gamma(t)))}{\mid \gamma(t)-\pi((\gamma(t)) \mid}\right)=0
$$

By L'Hospital's rule and the definability of $\gamma$ (which yields existence of the limits) we have

$$
\lim _{t \rightarrow 0} \frac{\gamma(t)-\pi(\gamma(t))}{|\gamma(t)-\pi(\gamma(t))|}=\lim _{t \rightarrow 0} \frac{\gamma^{\prime}(t)-\pi\left(\gamma^{\prime}(t)\right)}{\left|\gamma^{\prime}(t)-\pi\left(\gamma^{\prime}(t)\right)\right|} .
$$

It follows that:

$$
\lim _{t \rightarrow 0} P_{t}\left(\frac{\gamma^{\prime}(t)-\pi\left(\gamma^{\prime}(t)\right)}{\left|\gamma^{\prime}(t)-\pi\left(\gamma^{\prime}(t)\right)\right|}\right)=0
$$

Because $\gamma(t)$ is a definable map in a polynomially bounded o-minimal structure, there must be a positive real number $\varepsilon$ such that

$$
\frac{|\gamma(t)-\pi(\gamma(t))|}{t} \geq \varepsilon\left|\gamma^{\prime}(t)-\pi\left(\gamma^{\prime}(t)\right)\right| .
$$

(This is not true for an o-minimal structure which is not polynomially bounded, by Miller's dichotomy $[\mathbf{M}$. See the analysis of the example below. Or consider $\phi(t)=\exp (-C / t)$, which extends continuously at 0 by $\phi(0)=0$, and calculate $\phi^{\prime}(t)=C \phi(t) / t^{2}$ so that $\frac{\phi(t)}{t \phi^{\prime}(t)}=\frac{t}{C}$ which tends to 0 as $t$ tends to 0 .)

It is easy to see that if $\gamma$ is not tangent to $X$ at 0 then by $(a)$-regularity, the ratio test $(r)$ holds. Thus we may assume that $\gamma$ is tangent to $X$. Because also $\gamma$ is parametrised by arc-length, it follows that $\left|\gamma^{\prime}(t)\right|$ and $\left|\pi\left(\gamma^{\prime}(t)\right)\right|$ tend to 1 as $t$ tends to 0 .

Consequently, because $\gamma(t)$ lies on $Y$, and $\gamma$ is definable and thus may be assumed to be of class $C^{1}$ on $(0, \epsilon)$, it follows that $P_{t}\left(\gamma^{\prime}(t)\right)=0$, and we have:

$$
\begin{gathered}
R(\gamma(t))=\frac{\left|P_{t}\left(\pi\left(\gamma^{\prime}(t)\right)\right)\right||\gamma(t)|}{|\gamma(t)-\pi(\gamma(t))|\left|\pi\left(\gamma^{\prime}(t)\right)\right|} \approx \frac{\left|P_{t}\left(\gamma^{\prime}(t)-\pi\left(\gamma^{\prime}(t)\right)\right)\right| t}{|\gamma(t)-\pi(\gamma(t))|\left|\pi\left(\gamma^{\prime}(t)\right)\right|} \\
\approx \frac{\left|P_{t}\left(\gamma^{\prime}(t)-\pi\left(\gamma^{\prime}(t)\right)\right)\right| t}{|\gamma(t)-\pi(\gamma(t))|} \leq \frac{1}{\varepsilon} \frac{\left|P_{t}\left(\gamma^{\prime}(t)-\pi\left(\gamma^{\prime}(t)\right)\right)\right|}{\left|\gamma^{\prime}(t)-\pi\left(\gamma^{\prime}(t)\right)\right|},
\end{gathered}
$$

which tends to zero by (3.2). 
The converse implication, that $(r)$ implies $(b)$, this time for $Y$ of all dimensions, was also proved by Kuo $[\mathbf{K}]$. Actually Kuo treated the semi-analytic case as his paper predates the introduction of subanalytic sets. Verdier $[\mathbf{V e}$ confirmed that Kuo's proof works also for subanalytic stratifications. Here we show the new result that the implication is actually valid for definable sets in any o-minimal structure, not only for those which are polynomially bounded.

Proposition 3.2. Kuo's ratio test $(r)$ for $(Y, X)$ at $0 \in X$ implies Whitney's condition (b) at 0 for any o-minimal structure, and for $X$ of any dimension.

Proof. Fix a pair $(Y, X)$ satisfying $(r)$ at $0 \in X$. To prove that $(b)$ holds, take a definable $\gamma:[0, \varepsilon) \rightarrow X$ with $\gamma(0)=0$.

We may assume that $\gamma$ is parameterized by arc-length.

Then because $P_{t}\left(\gamma^{\prime}(t)\right) \equiv 0(\gamma(t)$ lies on $Y)$ we may write using (3.3):

$$
\begin{aligned}
& \left|P_{t}\left(\frac{\gamma^{\prime}(t)-\pi\left(\gamma^{\prime}(t)\right)}{\left|\gamma^{\prime}(t)-\pi\left(\gamma^{\prime}(t)\right)\right|}\right)\right|=\left|P_{t}\left(\frac{\pi\left(\gamma^{\prime}(t)\right)}{\left|\gamma^{\prime}(t)-\pi\left(\gamma^{\prime}(t)\right)\right|}\right)\right| \\
& \quad<\frac{t\left|P_{t}\left(\pi\left(\gamma^{\prime}(t)\right)\right)\right|}{|\gamma(t)-\pi(\gamma(t))|} \approx \frac{|\gamma(t)|\left|P_{t}\left(\pi\left(\gamma^{\prime}(t)\right)\right)\right|}{|\gamma(t)-\pi(\gamma(t))|\left|\pi\left(\gamma^{\prime}(t)\right)\right|} \leq R(\gamma(t)),
\end{aligned}
$$

using the mean-value theorem, and local monotonicity (due to definability and cell decomposition [DM], cf. the observation of Proposition 1.10 in [Loi]) of $\gamma^{\prime}$ to prove the strict inequality. But $R(\gamma(t))$ tends to zero in virtue of our hypothesis that $(r)$ holds. This shows that the angle between $\gamma^{\prime}-\pi\left(\gamma^{\prime}\right)$ and $T_{\gamma(t)} Y$ tends to zero as $t$ goes to zero. By (3.1), the angle between $\gamma-\pi(\gamma)$ and $T_{\gamma(t)} Y$ must tend to zero as well. This establishes Whitney's condition $(b)$ at 0 for the pair $(Y, X)$.

The previous proposition has as an immediate corollary that the Kuo-Verdier condition $(w)$ (which trivially implies $(r)$ ) also implies Whitney's condition $(b)$ for definable stratifications in any o-minimal structure, a result previously proved by Ta Lê Loi as Proposition 1.10 in Loi]. We remark that $(w)$ does not imply $(b)$ for general $C^{2}$ stratified sets - consider the topologist's sine curve in the plane, $S=\left\{y=\sin \frac{1}{x}: x>0\right\} \cup\{x=0,-1 \leq y \leq 1\}$, taking the two strata $\{x=0,-1 \leq$ $y \leq 1\}$ and its complement.

Let $(Y, X)$ be a pair of strata such that $X \subset \operatorname{cl}(X) \backslash Y$. As we may work with a coordinate system we shall identify $X$ with a neighborhood of the origin in $\mathbb{R}^{k} \times 0$. Denote by $\pi: \mathbb{R}^{n} \rightarrow \mathbb{R}^{k}$ the orthogonal projection.

Define for $t$ positive small enough:

$$
r(t):=\sup _{|\pi(y)|=t} \frac{\delta\left(X, T_{y} Y\right)}{|y-\pi(y)|} .
$$

It is clear that the Kuo-Verdier condition $(w)$ is equivalent to the boundedness of $r(t)$ for small $t$. Moreover the ratio test $(r)$ is equivalent to the conjunction of (a) and the property that $\operatorname{tr}(t)$ tends to zero as $t$ goes to zero.

Definition 3.3. Let $X$ and $Y$ be two strata with $X \subset \operatorname{cl}(X) \backslash Y$. We will say that $(Y, X)$ satisfies the condition $\left(r_{\int}\right)$ at $x \in X$ if $\int_{[0, \varepsilon]} r(t) d t<\infty$. 
We shall see that, combined with $(a)$-regularity, $\left(r_{\int}\right)$ implies local topological triviality, normal pseudo-flatness and the continuity of the density, in the case where the strata are definable.

DEFINITION 3.4. An $\alpha$-approximation of the identity is a homeomorphism $h: \mathbb{R}^{n} \times \mathbb{R}^{m} \rightarrow \mathbb{R}^{n} \times \mathbb{R}^{m}$ of type $h(x ; t)=\left(h_{t}(x) ; t\right)$ with:

$$
\begin{aligned}
\left|h_{t}(x)-x\right| & \lesssim \alpha(|x| ; t), \\
\left|h_{t}^{-1}(x)-x\right| & \lesssim \alpha(|x| ; t) .
\end{aligned}
$$

Given a definable set $A$ we set:

$$
\psi(A ; u)=\mathcal{H}^{k}(A \cap B(0 ; u)),
$$

where $k$ denotes the Hausdorff dimension of $A$ and $\mathcal{H}^{k}$ the $k$-dimensional Hausdorff measure. Recall that the density (or Lelong number) of $A$ at the origin is then defined as:

$$
\theta(A):=\lim _{u \rightarrow 0} \frac{\psi(A, u)}{\mu_{k} u^{k}}
$$

where $\mu_{k}$ stands for the volume of the unit ball in $\mathbb{R}^{k}$.

Theorem 3.5. (Theorem 4.5 of $\mathbf{\text { Va2 }}$ ) Let $A$ and $B$ be two definable (in some o-minimal structure) families of sets of dimension l of $\mathbb{R}^{n} \times \mathbb{R}^{m}$. Let $\alpha: \mathbb{R} \times \mathbb{R}^{m} \rightarrow$ $\mathbb{R}$ be a function with $\alpha(r, t) \leq \frac{r}{3}$, for all $t \in \mathbb{R}^{m}$, and let $h: A \rightarrow B$ be an $\alpha$ approximation of the identity and let $V$ be a compact subset of $\mathbb{R}^{m}$.

There is a constant $C$ such that for every $t \in V$ we have for $u>0$ small enough:

$$
\left|\psi\left(A_{t} ; u\right)-\psi\left(B_{t} ; u\right)\right| \leq C \alpha(u ; t) u^{l-1} .
$$

The next result is a generalisation of a theorem of Fukui and Paunescu [FP.

Proposition 3.6. Let $(A ; \Sigma)$ be a definable stratified set and assume that $Y:=$ $0 \times \mathbb{R}^{k}$ is a stratum. If all pairs of strata $\left(X, X^{\prime}\right) \in \Sigma \times \Sigma$ satisfy conditions (a) and $\left(r_{\rho}\right)$ then there exist neighbourhoods $U$ and $V$ of the origin in $\mathbb{R}^{n}$ and $\mathbb{R}^{k}$ and a topological trivialization

$$
h: A \cap U \rightarrow A_{0} \cap U \times V,
$$

$(x ; t) \mapsto\left(h_{t}(x) ; t\right)$ such that $h_{t}: \mathbb{R}^{n-k} \rightarrow \mathbb{R}^{n-k}$ is an $\alpha$-approximation of the identity with $\alpha(u ; t)=u \int_{0}^{t} r(s) d s$.

Proof. For simplicity, we will assume that $Y$ is one dimensional. We construct an isotopy by integration of vector fields as in Thom-Mather isotopy lemma [Ma.

Thanks to the $\left(r_{f}\right)$ condition the tangent spaces satisfy the following estimate:

$$
\delta\left(X ; T_{y} Y\right) \leq C r(|\pi(y)|) \cdot|y-\pi(y)|
$$

(where $\pi$ is the orthogonal projection onto $X$ ) in a sufficiently small neighborhood of the origin.

By standard arguments $\mathbf{V e}, \mathbf{M a}, \mathbf{O T 2}$ we may obtain a stratified unit vector field $v$ defined in a neighbourhood of the origin in $A$, tangent to $X$ and to the strata, smooth on every stratum, and satisfying $\pi(v)=\partial_{n}$ (where $\partial_{n}$ generates $X$ ) as well as:

$$
|v(y)-v(\pi(y))| \leq C r(|\pi(y)|) \cdot|y-\pi(y)|
$$


for some constant $C$.

Denote by $\phi$ the one-parameter group generated by this vector field. Let $\phi=$ $\left(\phi_{1} ; \phi_{2}\right) \in \mathbb{R}^{n-1} \times \mathbb{R}$; we also have by (3.4) (see OT3 Lemma 3.4) a positive constant $C$ such that:

$$
\left|\phi_{1}(q ;-t)-y\right| \leq C \int_{0}^{t} r(s) d s \cdot|y|
$$

for $q=(y, t) \in Y \subset \mathbb{R}^{n-1} \times \mathbb{R}$. Existence of integral curves is proved as in OT2 (see Lemma 3.3 of the latter article).

The desired trivialization in then given by $h_{t}(y):=\phi_{1}(q,-t)$ for $y \in A_{t}, q=$ $(y, t)$. By (3.5), it is an $\alpha$-approximation of the identity with $\alpha(u ; t)=u \int_{0}^{t} r(s) d s$.

The following theorem is a generalisation of Comte's theorem $\mathbf{C}$.

TheOREm 3.7. Let $(A ; \Sigma)$ be a definable stratified set and assume that $X:=$ $0 \times \mathbb{R}^{k}$ is a stratum. If all pairs of strata $\left(Y, Y^{\prime}\right) \in \Sigma \times \Sigma$ satisfy conditions $(a)$ and $\left(r_{\int}\right)$ then the density of $A$ is continuous along $X$.

Proof. Set

$$
\mathcal{A}:=\left\{\left(y ; t^{\prime} ; t\right) \in \mathbb{R}^{n-k} \times \mathbb{R}^{k} \times \mathbb{R}^{k}:\left(y ; t+t^{\prime}\right) \in Y\right\} .
$$

This defines a family of sets (parameterized by $t \in \mathbb{R}^{k}$ ) such that the germ of $\mathcal{A}_{t}$ at $t$ is the germ of $A$ at $\left(0_{\mathbb{R}^{n-k}} ; t\right)$. It is thus enough to prove that the function $t \mapsto \theta\left(\mathcal{A}_{t}\right)$ is continuous along the stratum $X$.

Apply Theorem 3.6 to get an $\alpha$ approximation of the identity $h: A \rightarrow A_{0} \times X$ with $\alpha(u ; t)=u \int_{0}^{t} r(s) d s$. Observe that the family $H_{t}\left(y, t^{\prime}\right):=h_{t^{\prime}}^{-1}\left(h_{t+t^{\prime}}(y)\right)$ maps $\mathcal{A}_{t}$ onto $\mathcal{A}_{0}$. We claim that it is an $\tilde{\alpha}$-approximation of the identity with $\tilde{\alpha}(u, t)=u \int_{[0, u+|t|]} r(s) d s$.

Take $\left(y, t^{\prime}\right)$ in $B(0, u)$. In particular $\left|t^{\prime}\right| \leq u$. Thus

$$
\left|h_{t+t^{\prime}}(y)-y\right| \lesssim u \int_{\left[0,\left|t^{\prime}\right|+|t|\right]} r(s) d s \leq u \int_{[0, u+|t|]} r(s) d s .
$$

In particular, this shows that $h_{t+t^{\prime}}(y)$ belongs to $B(0,2 u)$ (for $t$ and $t^{\prime}$ sufficiently small). Therefore, as $h$ is an $\alpha$-approximation of the identity:

$$
\left|h_{t^{\prime}}^{-1}\left(h_{t+t^{\prime}}(y)\right)-h_{t+t^{\prime}}(y)\right| \lesssim u \int_{\left[0, t^{\prime}\right]} r(s) d s \leq u \int_{[0, u+|t|]} r(s) d s .
$$

Together with the preceding estimate this implies the desired inequality. A similar computation holds for $H^{-1}$.

By theorem 3.5 we have for any $t$ and $u$ small enough:

$$
\left|\psi\left(\mathcal{A}_{t} ; u\right)-\psi\left(\mathcal{A}_{0} ; u\right)\right| \leq C u^{l} \int_{[0, u+|t|]} r(s) d s,
$$

where $l$ is the dimension of $A$ and $C$ is a positive constant. Dividing by $\mu_{k} u^{l}$ and passing to the limit as $u$ tends to zero we get:

$$
\left|\theta\left(\mathcal{A}_{t}\right)-\theta\left(\mathcal{A}_{0}\right)\right| \leq C \int_{[0,|t|]} r(s) d s
$$

which tends to zero as $t$ tends to zero. 
THEOREM 3.8. If a stratification, definable in some o-minimal structure (not necessarily polynomially bounded), satisfies both $(a)$ and $\left(r_{f}\right)$ then it satisfies conditions (npf) and $(n)$.

Proof. Fix a stratum $X$ of a $\left(r_{f}\right)$-regular stratification. Up to a coordinate system, we may assume that $X$ is $\{0\} \times \mathbb{R}^{k}, k=\operatorname{dim} X$. By Proposition 3.6, there exists a neighborhood $U$ of the origin (in $A$ ) and a topological trivialization

$$
h: A \rightarrow A_{0} \times V \text {, }
$$

$(y ; t) \mapsto\left(h_{t}(y) ; t\right)$ such that $h_{t}: \mathbb{R}^{n-k} \rightarrow \mathbb{R}^{n-k}$ is an $\alpha$ approximation of the identity with $\alpha(u ; t)=u \int_{[0,|t|]} r(s) d s$. This shows that the variation of the secant line $h_{t}(y) \pi\left(h_{t}(y)\right)$ (in the projective space) tends to zero as $t$ goes to zero.

The following proposition can be thought of as a generalisation of Pawłucki's theorem $\mathbf{P a}$, valid for subanalytic sets and without the $(n p f)$ criterion, which is a consequence of $(b)$ for subanalytic strata, by a theorem of Orro and Trotman OT3.

Proposition 3.9. Let $X$ and $Y$ be two strata which are definable in an ominimal structure. Assume that $\operatorname{dim} Y=\operatorname{dim} X+1$ and that $Y \cap B(0, \varepsilon)$ is connected for any $\varepsilon>0$ small enough. Then, $(Y, X)$ satisfies $(b)$ and $(n p f)$ if and only if $Y \cup X$ is a $C^{1}$ manifold with boundary.

Proof. (b) and (npf) are $C^{1}$ invariants. Hence, the if part is clear. Assume that these conditions hold and let us show that $Y \cup X$ is a $C^{1}$ manifold with boundary. We can identify $X$ with $\{0\} \times \mathbb{R}^{k}$.

We first show that at any point $x \in X, \lim _{y \rightarrow x} T_{y} Y$ exists. As the Whitney (b) condition implies the Whitney $(a)$ condition, we know that any limit of tangent space contains $T_{x} X$. By the Whitney $(b)$ condition, it also has to contain the limit of secant lines. Therefore, given a sequence $y_{i}$ tending to $x, \lim T_{y_{i}} Y$ (if it exists) is characterized by the limit in the projective space of $y_{i} \pi\left(y_{i}\right)$ (where $\pi$ is the orthogonal projection onto $X$ ).

Set $\gamma(y):=\left(\frac{y \pi(y)}{|y \pi(y)|}, y\right)$. for $y \in Y$, and $W:=\gamma(Y)$. As $W$ is a definable set of dimension $k+1$, we have:

$$
\operatorname{dim} c l(W) \backslash W \leq k .
$$

Moreover, if we set

$$
Z:=W \cap\left(S^{n-1} \times X\right)
$$

then, by the above inequality, $\operatorname{dim} Z \leq k$. Therefore, a generic fiber of the map $P: Z \rightarrow X$ defined by $(u, x) \mapsto x$ cannot have dimension bigger than 0 .

By $(n p f), P$ is an open map. Hence, $P^{-1}(0)$ must be of dimension 0 as well. As it is connected (since $Y$ is), it must be reduced to one single point. Hence, at any point $x$ of $X$ there is a unique limit of tangent space $\tau_{x}$, as claimed.

The limiting $l_{x}$ secant being unique at every point $x$ of $X$, it must vary continuously along $X$. Consequently, $\tau_{x}$ varies continuously as well. As a matter of fact, for a generic projection, the stratum $Y$ is the graph of a function whose derivative extends continuously. The couple $(\operatorname{cl}(Y), X)$ thus constitutes a $C^{1}$ manifold with boundary near the origin (due to topological triviality it must satisfy the frontier condition).

It follows that Theorem 3.8 admits the following corollaries. 
Corollary 3.10. Let $X$ and $Y$ be two (connected) strata which are definable in an o-minimal structure. Assume that $\operatorname{dim} Y=\operatorname{dim} X+1$ and that $(Y, X)$ satisfies (a) and $\left(r_{f}\right)$. Then $X \cup Y$ is a $C^{1}$ manifold with boundary.

Proof. By Proposition 3.2 ( $Y, X)$ satisfies the Whitney $(b)$ condition. Moreover, by Theorem 3.8, $X \cup Y$ is normally pseudo-flat. The result follows from the preceding proposition.

Corollary 3.11. Let $X$ and $Y$ be two (connected) strata which are definable in a polynomially bounded o-minimal structure. Assume that $\operatorname{dim} Y=\operatorname{dim} X+1$ and that $(Y, X)$ satisfies (b). Then $X \cup Y$ is a $C^{1}$ manifold with boundary.

Proof. By the proof of OT2 that $(b)$ implies $(n p f)$ in the subanalytic case, one may reduce to the case of 1-dimensional $X$. But then $(r)$ holds, by Proposition 3.1. By Theorem 3.8 $X \cup Y$ is normally pseudo-flat, because in the polynomially bounded case $(r)$ implies $\left(r_{f}\right)$. The result follows from the preceding proposition.

Corollary 3.11 is also a generalisation of Pawłucki's theorem [Pa].

\section{Pawłucki's example.}

In this section we study geometric properties of the closure $S_{g}$ of the graph in $\mathbf{R}^{3}$ of the function

$$
g(x, z)=\exp \left(\left(x^{2}+1\right) \ln (z)\right)=z^{x^{2}+1}, z>0 .
$$

and compare these with the geometric properties of the closure $S_{f}$ of the graph of a function previously studied by the first author and L. Wilson,

$$
f(x, z)=z-\frac{z}{\ln (z)} \ln \left(x+\sqrt{x^{2}+z^{2}}\right), z>0 .
$$

The natural stratification with two strata of $S_{f}$ was shown by the first author and Wilson in TW to be a counterexample in o-minimal geometry to several statements known to be true for sub-analytic sets, for example that Kuo's $(r)$ regular stratified sets $[\mathbf{K}$ are normally pseudo-flat (proved by Orro and the first author in OT3), and satisfy $\left(r^{*}\right)$-regularity (proved by Navarro Aznar and the first author in [NT]).

The set $S_{g}$ was used in a 1985 paper by Pawlucki in $[\mathbf{P a}$ as a counterexample to a possible generalisation of his useful and striking theorem concerning sub-analytic stratified sets with a smooth singular locus of codimension 1: such a stratified set is Whitney (b)-regular if and only if it is locally a finite union of $C^{1}$ manifolds with boundary (equal to the singular locus). For the natural two-strata stratification of $S_{g},(b)$-regularity holds and the set is homeomorphic to a closed half-plane, however $S_{g}$ is not a $C^{1}$ manifold with boundary. We shall show here that the graph of $g$ is also a counterexample to the geometric statements proved for sub-analytic sets in OT3, as well as having worse properties than the graph of $f$. For example although the graph of $g$ is Whitney $(b)$-regular over the 1-dimensional stratum $O x$, it does not satisfy Kuo's ratio test $(r)$, providing the first such example in $o$-minimal geometry. Kuo $[\mathbf{K}$ proved that no such example exists among semianalytic stratified sets, and the same proof is valid for subanalytic stratified sets. In Proposition 3.1 below we give a proof for definable sets in any polynomially 
bounded o-minimal structure. Moreover we show that $S_{g}$ can be used to define the first example of a definable stratification in an o-minimal structure which is Whitney (b)-regular but whose density (as defined by Kurdyka and Raby [KR], generalising the Lelong number $[\mathbf{L})$ is not continuous along strata.

Note that by Miller's dichotomy $[\mathbf{M}$ these examples exist in every o-minimal structure which is not polynomially bounded.

In $\mathbf{R}^{3}$ denote by $Y$ the graph of the function $f(x, z)$, for $z>0$ and $x$ and $z$ small, and denote by $W$ the graph of the function $g(x, z)$, for $z>0$ and $x$ and $z$ small. Denote the $x$-axis by $X$.

REMARK 4.1. $f(x, z)=-f(-x, z)$, i.e. $f$ is an odd function of $x$, while $g(x, z)$ is obviously an even function of $x$.

REMARK 4.2. $X \subset \bar{Y}$, because $\lim _{z \rightarrow 0} f(x, z)=0$.

Proof. Obviously,

$$
\lim _{z \rightarrow 0} z=0, \text { and } \quad \lim _{\mathrm{z} \rightarrow 0} \frac{1}{\ln \mathrm{z}}=0 .
$$

If $x>c>0$, then $\left|\ln \left(x+\sqrt{x^{2}+z^{2}}\right)\right|<|\ln (2 c)|$, so that

$$
\lim _{z \rightarrow 0} z \ln \left(x+\sqrt{x^{2}+z^{2}}\right)=0 .
$$

By remark 1 we do not need to study the case of $x<0$.

If both $x$ and $z$ tend to 0 , consider the two cases :

(i) $\frac{z}{x} \rightarrow 0$. Then

$$
\left|z \ln \left(x+\sqrt{x^{2}+z^{2}}\right)\right|<|z \ln (2 x)|<|x \ln (2 x)| \rightarrow 0 \quad \text { as } \quad \mathrm{x} \rightarrow 0,
$$

(ii) $\frac{x}{z}$ is bounded. Then

$$
\left|z \ln \left(x+\sqrt{x^{2}+z^{2}}\right)\right|<|z \ln z| \rightarrow 0 \quad \text { as } \quad \mathrm{z} \rightarrow 0 .
$$

Thus $X \subset \bar{Y}$. Clearly $\lim _{z \rightarrow 0} g(x, z)=0$, so that also $X \subset \bar{W}$.

Consider the closed stratified set $S_{f}$ with two strata $(Y, X)$, and the closed stratified set $S_{g}$ with two strata $(W, X)$. $(Y, X)$.

In [TW] the following five properties were shown to hold for $S_{f}$ stratified by

(1) $(n)$ and $(n p f)$ fail for $(Y, X)$ at $(0,0,0)$,

(2) Kuo's ratio test $(r)$ holds for $(Y, X)$ along $X$,

(3) Whitney's condition $(b)$ holds for $(Y, X)$ along $X$,

(4) $\left(b^{*}\right)$ and $\left(r^{*}\right)$ fail for $(Y, X)$ along $X$ at $(0,0,0)$,

(5) the density of $S_{f}$ is constant, hence continuous, along $X$.

Note that by (1) and (3), a general theorem of [T1 stating that $(w+\delta)$ implies $(n)$ and $(n p f)$, implies in turn that the Kuo-Verdier condition $(w)$ fails for $(Y, X)$ at $(0,0,0)$. Here $(\delta)$ refers to the weak Whitney condition introduced by Bekka and the first author (see [BT1] and [BT2]), which follows from (b) (as its name suggests).

Note also that (1) and (3) show that $S_{f}$ provides another (complicated) counterexample to Pawłucki's Theorem 0.1 above. 
We shall prove the following five properties for $S_{g}$ stratified by $(W, X)$.

(1) $(n)$ and $(n p f)$ fail for $(W, X)$ at $(0,0,0)$,

(2) Kuo's ratio test $(r)$ fails for $(W, X)$ along $X$ at $(0,0,0)$,

(3) Whitney's condition $(b)$ holds for $(W, X)$ along $X$,

(4) $\left(b^{*}\right)$ and $\left(r^{*}\right)$ fail for $(W, X)$ along $X$ at $(0,0,0)$,

(5) while the density of $S_{g}$ is constant along $X$ at $(0,0,0)$, the density of the 3-dimensional stratified set defined by the convex hull of $S_{g}$ and the upper half plane $\{y=0, z>0\}$ is not continuous along $X$ at $(0,0,0)$.

In particular Property 5 for $S_{g}$ shows that the theorems of Comte $[\mathrm{C}]$ and of the second author [Va1, Va2, proved for subanalytic sets, do not hold for general o-minimal structures. This is a surprise: it gives the first counterexample to continuity of the density along strata of Whitney regular stratified sets definable in some o-minimal structure. This answers negatively a question posed explicitly by the first author and L. Wilson on page 464 of [TW].

Property 1. (n) and (npf) fail for $S_{g}=W \cup X$ at $(0,0,0)$.

Proof.

We will show that the limits of secants from $(x, 0,0)$ to $(x, g(x, z), z)$ as $(x, z)$ tends to $\left(x_{0}, 0\right)$ are the straight lines which in the $(y, z)$-plane have equations

$y=0 \quad$ if $\quad x_{0}>0$

$y=\sigma z$ for all $\sigma \in[0,1] \quad$ if $\quad x_{0}=0$

$y=0 \quad$ if $\quad x_{0}<0$.

However, for the secants from $(0,0,0)$ to $(0, f(0, z), z)$ as $z$ tends to 0 , the limiting secant is $y=z$. Hence $(n)$ fails (the tangent cone to $C_{0}\left(S_{0}\right)$ does not equal the fibre at 0 of the normal cone). Moreover $(n p f)$ fails since for $x_{0} \neq 0$ the fibre at $x_{0}$ of the normal cone is 0 -dimensional, while the fibre at 0 is 1 -dimensional.

Proof of (1.1). First observe that, for all $0<z<1$, the secant from $(0,0,0)$ to $(0, g(0, z), z)$ has slope

$$
\frac{g(0, z)}{z}=1 .
$$

Take $x_{0}>0$ and let $(x, z)$ tend to $\left(x_{0}, 0\right)$. The slope of the secant from $(x, 0,0)$ to $(x, g(x, z), z)$ is

$$
\frac{g(x, z)}{z}=z^{x^{2}}=\exp \left(x^{2} \ln z\right)
$$

which tends to 0 as $z$ tends to 0 and $x$ tends to $x_{0}$.

By symmetry (Remark 1 ), when $x_{0}<0$ the limiting slope is also 0 .

Now suppose $(x, z)$ tends to $(0,0)$.

By symmetry (Remark 1 again) it will be enough to study the case $x>0$ and to show that all the values $\sigma \in[0,1]$ are realised. So we must show that the limits of $z^{x^{2}}$ take all values in $[0,1]$ as $x$ and $z$ tend to 0 when $x>0$.

Let $\sigma \in(0,1)$.

On the curve $z=\exp \left(\frac{\ln \sigma}{x^{2}}\right)$, i.e. $x^{2} \ln z=\ln \sigma, \exp \left(x^{2} \ln z\right)=\sigma$ so in particular the limit as $(x, z)$ tends to $(0,0)$ is $\sigma$.

On the curve $z=\exp \left(\frac{-1}{x^{3}}\right), \exp \left(x^{2} \ln z\right)=\exp \left(\frac{-1}{x}\right)$ with limit 0 as $x$ tends to 0 .

This completes the proof of (1.1), and hence the proof of Property 1.

Next we shall study Property 2, which is Kuo's ratio test $(r)$. We show that $(r)$ fails for $(W, X)$ at $(0,0,0)$, the condition failing along flat curves of the form $z=\exp \left(\frac{-C}{x^{2}}\right)$. That such an example exists is surprising, because we shall see 
below that Property 3 - Whitney's condition $(b)$ - does hold, and this is enough to ensure $(r)$ in the subanalytic case along strata of dimension one, as was first shown in 1970 by Tzee-Char Kuo $[\mathbf{K}$. Along strata of higher dimension it is not the case that $(r)$ follows from $(b)$-regularity as was illustrated by the semi-algebraic examples constructed by the first author during the Nordic Summer School at Oslo in August 1976 [T1. Real algebraic examples were given in the first author's 1977 Warwick thesis [T2 and can be found with other real algebraic examples in a joint paper by the first author with Brodersen $[\mathbf{B r T}]$. The systematic calculations of (b)-regularity by the first author in his 1977 thesis [T2, completed in [T3 and of $(w)$-regularity in the 1996 thesis of Noirel $[\mathbf{N}]$ provide infinitely many real algebraic examples.

Property 2. ( $r)$ fails for the pair of strata $(W, X)$ at $(0,0,0)$.

Proof.

Recall that Kuo's ratio test $(r)$ holds when

$$
R(x, y, z)=\frac{|(x, y, z)| \cdot \delta\left(T_{(x, 0,0)} X, T_{(x, y, z)} W\right)}{\|(y, z)\|} \rightarrow 0
$$

as $(x, y, z)$ tends to $(0,0,0)$ on $W$.

Now, $\quad \delta\left(T_{(x, 0,0)} X, T_{(x, y, z)} W\right)=\frac{\left|\frac{\partial g}{\partial x}\right|}{\left\|\left(\frac{\partial g}{\partial x},-1, \frac{\partial g}{\partial z}\right)\right\|}<\left|\frac{\partial g}{\partial x}\right|$.

Calculating, using $g=\exp \left(\left(x^{2}+1\right) \ln z\right)$,

$$
\left|\frac{\partial g}{\partial x}\right|=\left|2 x \cdot \ln z \cdot \exp \left(\left(x^{2}+1\right) \ln z\right)\right|=\left|2 x \cdot z \cdot \ln z \cdot \exp \left(x^{2} \cdot \ln z\right)\right|,
$$

which tends to zero as $z$ tends to 0 , because $z \cdot \ln z$ tends to 0 as $z$ tends to 0 , while $\exp \left(x^{2} \cdot \ln z\right)$ is bounded above by 1 as $x$ and $z$ tend to 0 . Further,

$$
\left|\frac{\partial g}{\partial z}\right|=\exp \left(x^{2} \ln z\right)
$$

which is bounded between 0 and 1 as $x$ and $z$ both tend to zero.

$$
\text { Thus, } \quad \begin{aligned}
R(x, y, z) \approx & \frac{\left|\frac{\partial g}{\partial x}\right| \cdot||(x, y, z)||}{\|(y, z)||} \approx \frac{\left|\frac{\partial g}{\partial x}\right|}{|z|} \cdot \sqrt{x^{2}+z^{2}} \\
& \approx\left|x \cdot z \cdot \ln z \cdot \exp \left(x^{2} \ln z\right) \cdot \frac{\sqrt{x^{2}+z^{2}}}{z}\right| \\
& =\left|x \cdot \ln z \cdot \exp \left(x^{2} \ln z\right) \cdot \sqrt{x^{2}+z^{2}}\right| .
\end{aligned}
$$

We need to check whether this tends to zero as $z$ tends to 0 .

If $z$ dominates $x$, then because $z \cdot \ln z$ tends to zero as $z$ tends to zero, we can deduce that $R(x, y, z)$ also goes to zero.

There remains the case where $x$ dominates $z$ to consider. In this case $R(x, y, z)$ will be equivalent to $x^{2} \ln z \exp \left(x^{2} \ln z\right)$. Because $x^{2} \ln z$ has values running from 0 to $-\infty$ as $x$ and $z$ tend to zero, we need to study the function $w \exp (w)$ for $w \in]-\infty, 0]$. This takes the value 0 when $w=0$, and also tends to 0 as $w$ tends to $-\infty$. However we can also choose $x$ and $z$ tending to 0 so that $w=x^{2} \ln z$ tends to a constant $-C, C>0$. In particular one may find $x$ and $z$ tending to zero on 
the curve $x^{2} \ln z=-C$, i.e. $z=\exp \left(-C / x^{2}\right)$. Such a curve is flat, tangent to the $x$-axis, and the associated curve

$$
\begin{aligned}
\left(x, \exp \left(\left(x^{2}+1\right) \ln z\right), z\right) & =\left(x, \exp \left(-C\left(x^{2}+1\right) / x^{2}\right), \exp \left(-C / x^{2}\right)\right) \\
& =\left(x, e^{-C} e^{-C / x^{2}}, e^{-C / x^{2}}\right)
\end{aligned}
$$

lies on $W$. The limit of $R$ restricted to such a curve is clearly equivalent to $-C \exp (-C)$, so that the ratio test $(r)$ will fail to hold at $(0,0,0)$ for the pair $(W, X)$.

Note. Condition $(a)$ holds. As above, $d\left(T_{(x, 0,0)} X, T_{(x, y, z)} W\right)<\left|\frac{\partial g}{\partial x}\right|$, and we saw that $\left|\frac{\partial g}{\partial x}\right|$ tends to 0 as $z$ tends to 0 , giving $(a)$-regularity.

By the main theorem of $\mathbf{O T 2}$, the condition $\left(r^{e}\right)$ (defined in $\mathbf{O T 2}$ ) must fail for $(W, X)$ at the origin, because $(a)$ holds, while $(n)$ and $(n p f)$ fail.

Property 3. (b) holds for $(W, X)$ at $(0,0,0)$.

Proof. We have just seen that $(a)$ holds. Thus we need only prove that $\left(b^{\pi}\right)$ holds.

By remark 1 , we need only treat the case $x \geq 0$.

Suppose $0<z<1$, and $0 \leq x$, for $x$ small.

To prove that Whitney $\left(b^{\pi}\right)$ holds at $(0,0,0)$ we must show that

$$
Q(x, z)=\frac{-y+z g_{z}}{\|(y, z)\| \cdot\left\|\left(g_{x},-1, g_{z}\right)\right\|}
$$

tends to zero as $(x, z)$ tends to $(0,0)$.

Now $Q(x, z)=\frac{-y+z^{x^{2}+1}\left(x^{2}+1\right)}{\|(y, z)\| \cdot\|\cdot\|\left(g_{x},-1, g_{z}\right) \|}=\frac{x^{2} z^{x^{2}+1}}{\|(y, z)\| \cdot\left\|\left(g_{x},-1, g_{z}\right)\right\|}$ which is equivalent to $x^{2} z^{x^{2}}$, which tends to zero as $x$ and $z$ tend to 0 .

This implies that $\left(b^{\pi}\right)$ holds, and hence that $(b)$ holds for $(W, X)$ on a neighborhood of $(0,0,0)$ in $X$.

Property 4. $\left(b^{*}\right)$ and $\left(r^{*}\right)$ fail for $(W, X)$ at $(0,0,0)$.

Proof. We intersect $W$ with planes $\{y=a z, 0<a<1\}$ to obtain

$$
a z=\exp \left(\left(x^{2}+1\right) \ln z\right)=z z^{x^{2}},
$$

which becomes

$$
a=\exp \left(x^{2} \ln z\right) .
$$

Hence $\ln a=x^{2} \ln z, x^{2}=\frac{\ln a}{\ln z}$, and $z=e^{\frac{\ln a}{x^{2}}}$.

This curve in the $x z$-plane passes through $(0,0)$ if $0<a<1$, since $Y \cap\{y=a z\}$ contains curves passing through $(0,0,0)$. It follows that $(W \cap\{y=a z\}, X)$ cannot be $(b)$-regular, and then by definition $(W, X)$ is not $\left(b_{\operatorname{cod} 1}\right)$-regular and $\left(b^{*}\right)$ fails. Note that $\left(a_{\operatorname{cod} 1}\right)$ holds for $(W, X)$, as does $(a)$, and thus $\left(a^{*}\right)$ holds. However $\left(r^{*}\right)$ fails because $(r)$ fails at $(0,0,0)$.

Property 5. While the density of $S_{g}$ is continuous along $X$ at $(0,0,0)$, the density of the 3 dimensional stratified set $K_{g}$, defined by the convex hull of the union of $S_{g}$ with the upper half plane $y=0, z>0$, is not continuous along $X$ at $(0,0,0)$.

Proof. By the proof of Property 1, together with $(a)$-regularity, we see that the pure tangent cone at each $\left(x_{0}, 0,0\right)$, where $x_{0} \neq 0$, is $\{y=0, z \geq 0\}$. The 
density of $S_{g}$ at such points is thus $1 / 2$ according to the definition and results of Kurdyka and Raby [KR], while the density of $K_{g}$ at such points on $X$ is 0 .

The set of limiting secants to $S_{g}$ from the origin $(0,0,0)$ (defining the tangent cone to $S_{g}$ at $(0,0,0)$ ) is precisely $\{y=z\}$, so that by the formula for the density of Kurdyka and Raby in terms of the pure tangent cone [KR, we see that the density at the origin of $S_{g}$ is $1 / 2$, while the density at the origin of $K_{g}$ is $1 / 8$ (the area $\pi / 8$ of the part of the sector between $y=0$ and $y=z$ inside the unit ball centred at the origin, divided by the area $\pi$ of the unit disk). It follows that the density of $S_{g}$ is constant along $X$, and the density of $K_{g}$ along $X$ jumps at $(0,0,0)$.

REMARK 4.3. The natural stratification of $K_{g}$ by dimension is Whitney $(b)$ regular, with one connected stratum of dimension 3 , two strata of dimension 2 the graph of $g$ and the upper half-plane $\{y=0, z>0\}$ - and a single stratum of dimension 1 , namely the $x$-axis. Using the same technique as above to obtain a 3-dimensional stratified set $K_{f}$ equal to the convex hull of the union of $S_{f}$ and the upper half-plane $\{y=0, z>0\}$, we find that its natural stratification by dimension has two strata of dimension 1 , namely the $x$-axis and the half-line $\{y=0, x=0\}$, so forcing the origin to be a stratum. Hence the density of $K_{f}$ is continuous along strata.

\section{References}

[BT1] K. Bekka and D. Trotman, Propriétés métriques de familles $\Phi$-radiales de sous-variétés différentiables, C. R. Acad. Sci. Paris, série I, t. 308 (1987), 389-392.

[BT2] K. Bekka and D. Trotman, Weakly Whitney stratified sets, Real and complex singularities (Proceedings, Sao Carlos 1998, edited by J.W. Bruce and F. Tari), Chapman and Hall/CRC (2000), 1-15.

[Be] A. Bernig, Curvature bounds on subanalytic spaces, preprint, 2003.

[BrT] H. Brodersen and D. Trotman, Whitney (b)-regularity is strictly weaker than Kuo's ratio test for real algebraic stratifications, Math. Scand., 45 (1979), 27-34.

[CK] P. Chossat and M. Koenig, Characterization of bifurcations for vector fields which are equivariant under the action of a compact Lie group, C. R. Acad. Sci. Paris, série I, t. 318 (1994), 31-36.

[C] G. Comte, Equisingularité réelle : nombres de Lelong et images polaires, Ann. Sci. École Norm. Sup., Paris (4) 33 (2000), 757-788.

[CM] G. Comte and M. Merle, Equisingularité réelle. II. Invariants locaux et conditions de régularité, Ann. Sci. École Norm. Sup., Paris (4) 41 (2008), 221-269.

$[\mathrm{DM}]$ L. van den Dries and C. Miller, Geometric categories and o-minimal structures, Duke Math. Journal 84 (1996), 497-540.

[D] L. van den Dries, Tame Topology and o-minimal structures, Cambridge University Press, London Mathematical Society Lecture Note Series 248, 1998.

[FFW] M. Ferrarotti, E. Fortuna and L. Wilson, Approximation of subanalytic sets by normal cones, Bulletin of the London Math. Soc. 39 (2007), 247-254.

[FP] T. Fukui and L. Paunescu, Stratification theory from the weighted point of view, Canadian J. Math. 53 (2001), 73-97.

[HM1] J.-P. Henry and M. Merle, Limites de normales, conditions de Whitney et éclatement d'Hironaka, Proc. Sympos. Pure Math. 40 (Amer. Math. Soc, Providence, R.I., 1983), Part 1, 575-584.

[HM2] J.-P. Henry and M. Merle, Stratifications de Whitney d'un ensemble sous-analytique, C. R. Acad. Sci. Paris, série I, t. 308 (1989), 357-360.

$[\mathrm{H}] \quad$ H. Hironaka, Normal cones in analytic Whitney stratifications, Inst. Hautes Études Sci. Publ. Math. 36 (1969), 127-138.

[J] A. Jeddi, Preuve d'une conjecture de Palamodov, Topology 41 (2002), 271-306. 
[JTV] D. Juniati, D. Trotman and G. Valette, Lipschitz stratifications and generic wings, Journal of the London Math. Soc. (2) 68 (2003), 133-147.

[K] T.-C. Kuo, The ratio test for analytic Whitney stratifications, Proceedings of the Liverpool Singularities Symposium I (C.T.C. Wall, ed.), Springer Lecture Notes in Math. 192 (1971), 141-149.

[KR] K. Kurdyka and G. Raby, Densité des ensembles sous-analytiques, Ann. Inst. Fourier 39 (1989), 735-771.

[KT] M. Kwieciński and D. Trotman, Scribbling continua in $\mathbf{R}^{n}$ and constructing singularities with prescribed Nash fibre and tangent cone, Topology and its Applications 64 (1995), 177-189.

[L] P. Lelong, Intégration sur un ensemble analytique complexe, Bull. Soc. Math. France 85 (1957), 239-262.

[Loi] T. L. Loi, Verdier and strict Thom stratifications in o-minimal structures, Illinois J. Math. 42 (1998), 347-356.

[Loj] S. Łojasiewicz, Théorème de Pawtucki. La formule de Stokes sous-analytique, Geometry Seminars, 1988-1991 (Bologna, 1988-1991), Univ. Stud. Bologna, Bologna (1991), 79-82.

[Ma] J. Mather, Notes on topological stability, Harvard University, 1970.

[Me] M. Merle, Variétés polaires, stratifications de Whitney et classes de Chern des espaces analytiques complexes, Séminaire Bourbaki, 25 (1982-1983), Exposé No. 600, 14 pages.

[M] C. Miller, Exponentiation is hard to avoid, Proc. Amer. Math. Soc. 122 (1994), 257-259.

[NT] V. Navarro Aznar and D. Trotman, Whitney regularity and generic wings, Annales Inst. Fourier, Grenoble 31 (1981), 87-111.

[NV] N. Nguyen and G. Valette, Whitney stratifications and the continuity of local Lipschitz Killing curvatures, arXiv:1507.01262.

[N] L. Noirel, Plongements sous-analytiques d'espaces stratifiés de Thom-Mather, University of Provence thesis, 1996.

[OP] P. Orro and F. Pelletier, Indice local pour des ensembles symétriques par arcs, Math. Z. 233 (2000), 817-828.

[OT1] P. Orro and D. Trotman, On the regular stratifications and conormal structure of subanalytic sets, Bull. London Math. Soc. 18 (1986), 185-191.

[OT2] P. Orro and D. Trotman, Cône normal à une stratification régulière, Seminari Geometria 1998-99, Università degli Studi Bologna, t. 12 (2000), 169-175.

[OT3] P. Orro and D. Trotman, Cône normal et régularités de Kuo-Verdier, Bull. Soc. Math. France 130 (2002), 71-85.

[Pa] W. Pawłucki, Quasi-regular Boundary and Stokes' formula for a sub-analytic leaf, Seminar on Deformations, Łodz-Warsaw 1981-83, Springer Lecture Notes in Math. 1165 (1985), 235-252.

[Te] B. Teissier, Introduction to equisingularity problems, A. M. S. Algebraic Geometry Symposium, Arcata 1974, Providence; Rhode Island (1975), 593-632.

[Te1] B. Teissier, Variétés polaires II: multiplicités polaires, sections planes et conditions de Whitney, Algebraic Geometry, La Rabida 1981, Lecture Notes in Math. 961 (Springer, Berlin, 1982), 314-491.

[Te2] B. Teissier, Sur la classification des singularités des espaces analytiques complexes, Proc. of the International Congress of Mathematicians, Vol. 1, 2 (Warsaw, 1983), PWN, Warsaw (1984) 763-781.

[T1] D. Trotman, Counterexamples in stratification theory: two discordant horns, Real and complex singularities (ed. P. Holm), Oslo 1976, Nordic Summer School, Sijthoff and Noordhoff, 1977, 679-686.

[T2] D. Trotman, Whitney stratifications: faults and detectors, Warwick University thesis, 1977 .

[T3] D. Trotman, On the canonical Whitney stratifications of algebraic hypersurfaces, Séminaire sur la Géométrie Algébrique Réelle (dir. J.-J. Risler), Publ. Math. Univ. Paris VII, 24 (1987), vol. 1, 123-152.

[TW] D. Trotman and L. Wilson, $(r)$ does not imply $(n)$ or $(n p f)$ for definable sets in non polynomially bounded o-minimal structures, Advanced Studies in Pure Mathematics $\mathbf{4 3}$ (2006), 463-475.

[Va1] G. Valette, Détermination et stabilité du type métrique des singularités, University of Provence thesis, 2003. 
[Va2] G. Valette, Volume, Whitney conditions and Lelong number, Ann. Polon. Math. 93 (2008), 1-16.

[Va3] G. Valette, $L^{\infty}$ cohomology is intersection cohomology, Advances in Mathematics 231 (2012), 1818-1842.

[Va4] G. Valette, Stokes' formula for stratified forms, Ann. Polon. Math. 114 (2015), 197-206.

[Ve] J.-L. Verdier, Stratifications de Whitney et théorème de Bertini-Sard, Inventiones Math. 36 (1976), 295-312.

Aix Marseille Univ., CNRS, Centrale Marseille, Institut Mathématique de MarSeille, 13453 Marseille, France.

E-mail address: david.trotman@univ-amu.fr

Instytut Matematyczny PAN, ul. Św. Tomasza 30, 31-027 Kraków, Poland

E-mail address: gvalette@impan.pl 